\title{
PERFIL DAS COMPLICAÇÕES APÓS APENDICECTOMIA EM UM HOSPITAL DE EMERGÊNCIA
}

\section{Post-appendectomy complication profiling in an emergency hospital}

\author{
Diogo Braga de Albuquerque NUTELS, Ana Catarina Gadelha de ANDRADE, Amaurí Clemente da ROCHA
}

\section{ABCDDV/548}

\begin{abstract}
Nutels DBA, Andrade ACG, Rocha AC. Perfil das complicações após apendicectomia em um hospital de emergência. ABCD Arq Bras Cir Dig 2007;20(3):146-9.

RESUMO - Racional - A apendicite aguda é a lesão inflamatória cirúrgica aguda mais freqüente da cavidade abdominal. Objetivo - Analisar as complicações pós-operatórias mais freqüentes em apendicectomias. Método - Análise retrospectiva de pacientes submetidos à apendicectomias na Unidade de Emergência Dr. Armando Lages, no período de julho de 2005 a julho de 2006, aplicando-se um protocolo de pesquisa. Resultados - Do total de 107 pacientes apendicectomizados, ocorreram complicações em 17\%. Treze pacientes (54\%) evoluíram com infecção da ferida operatória, seguida por abscesso de parede em $12,5 \%$ e pela peritonite em $8 \%$. Houve $0,9 \%$ de reoperações e $0,9 \%$ de óbitos. Dos apendicectomizados $5,6 \%$ pertenciam à faixa etária pré-escolar, e destes $33 \%$ evoluíram com complicações. Nos escolares e adolescentes ocorreram $10 \%$ de complicações e nos adultos jovens, $20 \%$. Adultos de meia idade e idosos, tiveram $66 \%$ de complicações. Dos que evoluíram em até 24 horas de doença, 17,2\% tiveram algum tipo de complicação no pós-operatório; entre 1 e 3 dias, $15 \%$ e os com mais de 4 dias, $57,2 \%$. Conclusão - Complicações nas apendicectomias foram mais freqüentes nos extremos de idade, quando houve atraso no diagnóstico e tratamento, e nas fases mais tardias da apendi DESCRITORES - Apendicite. Apendicectomia. Complicações pós-operatórias.
\end{abstract}

\section{INTRODUÇÃO}

A apendicite aguda é a doença inflamatória abdominal cirúrgica de maior freqüência que acomete, principalmente, indivíduos jovens em fase produtiva da vida ${ }^{1}$.

Essa doença tem sido atribuída à obstrução mecânica, à dieta inadequada de fibras e à susceptibilidade familiar $^{2,4,5,7,8,9}$. Outros fatores relacionados à estrutura populacional, condições socioeconômicas e patógenos bacterianos, virais ou parasitários, também estão envolvidos na etiopatogenia dessa afecção. Entretanto, sua causa e epidemiologia ainda permanecem pouco compreendidas $^{2,3,6}$.

Duas teorias etiopatogênicas da apendicite aguda são as mais aceitas: a da disseminação bacteriana, levando ao processo inflamatório do apêndice cecal ${ }^{10} \mathrm{e}$ a da obstrução do lume apendicular, desencadeando a estase, hipersecreção e proliferação das bactérias já existentes nele ${ }^{11,12}$.

O diagnóstico é essencialmente clínico. A riqueza dos sintomas e sinais pesquisados, a anamnese e o exame físico bem realizado, firma-o na maioria dos casos ${ }^{13}$.

Esta doença, geralmente, apresenta evolução satis-

Trabalho realizado na Unidade de Emergência Dr. Armando Lages, Maceió-AL, Brasil.

Endereço para correspondência: Diogo Braga de Albuquerque Nutels, e-mail: diogonutels@yahoo.com.br fatória, desde que seja realizado o tratamento cirúrgico; entretanto, em casos onde há dificuldade ou retardo no diagnóstico, sua evolução para uma fase tardia, possibilita o aparecimento de complicações graves como a peritonite generalizada, a pileflebite e o abscesso hepático ${ }^{14}$.

As complicações na evolução da apendicite aguda correm paralelamente à gravidade da lesão apendicular ${ }^{15}$.

O tratamento é cirúrgico, sendo a incisão mais utilizada a de McBurney, porém nos casos duvidosos ou mais avançados é preferível a mediana ${ }^{13}$.

O objetivo deste trabalho foi analisar as complicações pós-operatórias mais freqüentes em apendicectomias.

\section{MÉTODO}

Este estudo retrospectivo foi realizado na Unidade de Emergência Dr. Armando Lages, em Maceió, AL, Brasil, coletando-se os dados a partir da análise de 107 prontuários de pacientes submetidos à apendicectomia no período de julho de 2005 a julho de 2006 escolhidos aleatoriamente. Observou-se a freqüência de complicações pós-operatórias, o número de reintervenções e óbitos, distribuição das complicações pós-operatórias de acordo com o sexo e a faixa etária, o período de evolução da doença (do início dos sintomas ao ato cirúrgico) e sua relação com as complicações pós-operatórias, bem como os achados operatórios (fases da apendicite aguda) e sua 
associação com as complicações pós-operatórias.

O protocolo de pesquisa constava da identificação do paciente (idade, sexo, estado civil, ocupação, naturalidade), principais sinais e sintomas, tempo decorrido do início do quadro e momento da operação, achados operatórios, tipo de incisão, técnica operatória utilizada, tipo de antibiótico usado, complicações encontradas no pós-operatório e em que momento surgiram essas complicações, reoperações e óbito.

Os dados foram agrupados em banco de dados do programa Epi-InFo, versão 6.2 e posteriormente, os resultados foram analisados quantitativamente, por tipo e freqüência dos achados. Foi realizada a análise univariada e multivariada, utilizando-se o coeficiente de determinação (R2), sendo o $P \leq 0,05$ determinando significância estatística.

\section{RESULTADOS}

Do total de 107 pacientes apendicectomizados, ocorreram complicações em $17 \%$. Treze pacientes (54\%) evoluíram com infecção da ferida operatória, que representou a complicação mais freqüente, seguida pelo abscesso de parede em $12,5 \%$ e pela peritonite $8 \%$. Foi encontrado apenas um caso de cada uma das seguintes complicações: abscesso intracavitário, fístula cecal, fístula transdiafragmática, pneumonia, empiema e sepse. Houve ainda, 0,9\% de reoperações e $0,9 \%$ de óbitos (Figura 1).

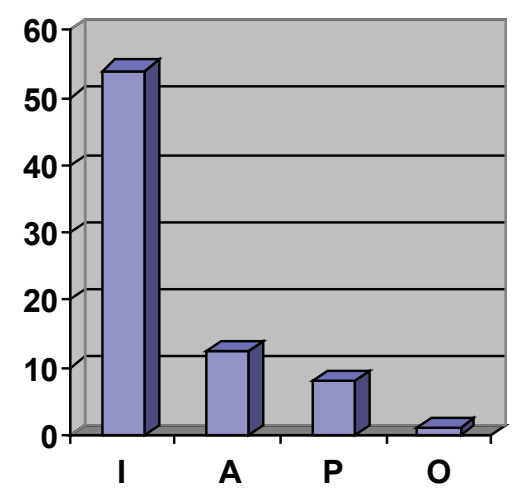

$\square$ Complicação

FIGURA 1 - Freqüência das complicações após apendicectomias. I - infecção de ferida operatória; A - abcesso de parede; $\mathrm{P}$ - peritonite; $\mathrm{O}$ - outros.

Eram $74 \%$ do sexo masculino e $22 \%$ destes complicaram, enquanto que dentre as mulheres (26\%), apenas $4 \%$ evoluíram com complicações no pós-operatório (Figura 2). Quanto à faixa etária, não foi observado apendicite aguda em menores de 1 ano. Dos apendicectomizados, 5,6\% pertenciam a faixa etária pré-escolar ( 1 a 4 anos), e destes $33 \%$ evoluíram com complicações. Nos escolares e adolescentes (5 a 19 anos), 51,4\% dos casos, 10\% complicaram. Adultos jovens (20 a 49 anos), 40,2\% dos casos, 20\% complicaram. Por último, adultos de meia idade e idosos (maiores que 50 anos) corresponderam a 2,8\% dos pacientes e destes, $66 \%$ foram acometidos por complicações (Figura 3).

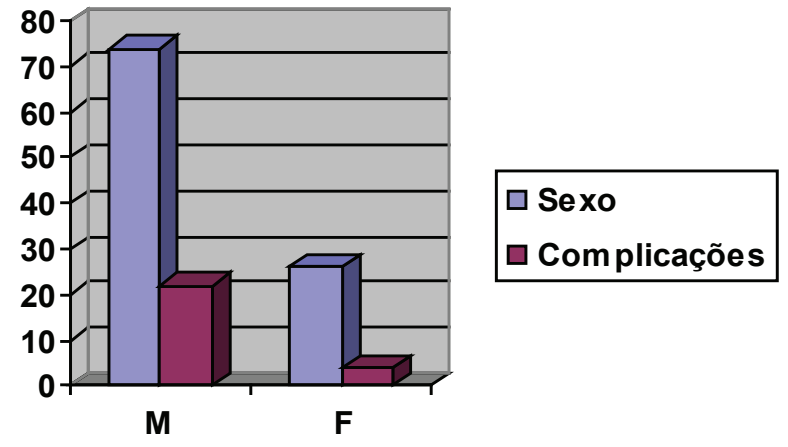

FIGURA 2 - Prevalência de indivíduos apendicetomizados e incidência de complicações por sexo

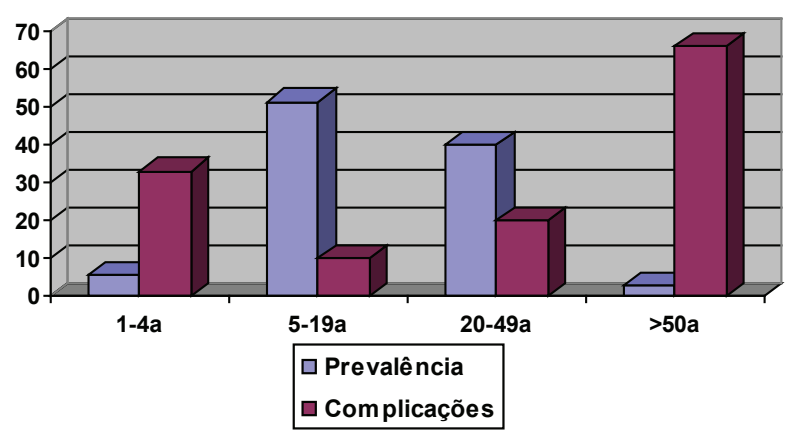

FIGURA 3 - Prevalência de indivíduos apendicectomizados e incidência de complicações por idade

Dos que evoluíram em até 24 horas de doença (31,5\% dos casos estudados), $17,2 \%$ tiveram algum tipo de complicação no pós-operatório. Entre 1 e 3 dias $(43,5 \%)$ de evolução, $15 \%$ complicaram, e por fim, os com mais de 4 dias $(22,8 \%$ ), obtiveram complicações em $57,2 \%$ dos casos (Figura 4).

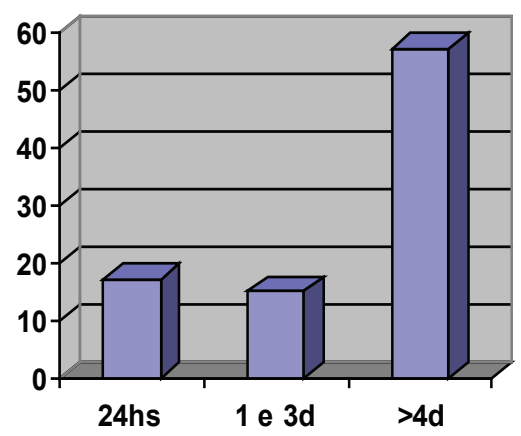

$\square$ Complicação

FIGURA 4 - Freqüência de complicações após apendicectomia em relação ao período de evolução da doença

Foram encontrados apêndices inflamados (fase I) em $23,5 \%$ das apendicectomias, $27 \%$ supurados (fase II), $22,5 \%$ necrosados (fase III) e $27 \%$ perfurados (fase IV). O percentual de complicações pós-operatórias nas fases I e II foi de $11 \%$. Já em estágios mais avançados, ou seja, fases III e IV, foi de 76\% (Figura 5). 


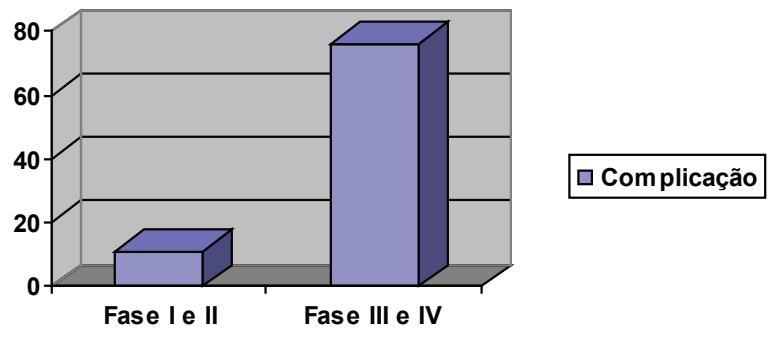

FIGURA 5 - Incidência de complicações após apendicectomias de acordo com o achado intra-operatório

\section{DISCUSSÃO}

É prudente o uso de antibióticos no pré-operatório, no entanto a continuação da terapêutica vai depender dos achados operatórios de abscesso ou perfuração livre ${ }^{16}$. Já foi relatado que o uso de metronidazol e gentamicina, no lugar de cloranfenicol, reduziu significativamente a incidência de complicações infecciosas no pós-operatório de apendicite supurada (de $31 \%$ para $5 \%)^{17}$.

As complicações locais mais freqüentes referidas na literatura são os abscesso de parede, abscessos residuais, obstrução intestinal, fistula fecal, evisceração, eventração, peritonites e hemorragia ${ }^{16}$. Outras complicações gerais comuns a qualquer celiotomia são as infecções respiratórias, urinárias, cardíacas, hematomas, hemorragias subcutâneas e infecções hospitalares, em geral, flebotromboses e tromboflebites sépticas ${ }^{13}$. Este estudo confirma esses achados.

As complicações pós-operatórias mais comuns da apendicectomia estão relacionadas com o grau de inflamação apendicular. É importante levar em consideração o tempo decorrido do início do quadro e o momento da operação ${ }^{16}$. Em estudo com pacientes com apendicite aguda submetidos à apendicectomias correlacionando a freqüência de infecções da ferida operatória com o tempo de evolução da doença, foi encontrado infecção em 1,7\% em pacientes com menos de 24 horas de evolução, $11 \%$ com 1 a 3 dias e $78.9 \%$ nos pacientes com mais de 4 dias $^{18}$, dados esses semelhantes aos aqui encontrados.

Em outro estudo em que os pacientes com apendicite aguda que foram operados e tiveram o apêndice extraído de forma intacta, foi observado índice de complicações de $5 \%$. No entanto, dos pacientes que apresentaram o apêndice perfurado ou gangrenoso, $30 \%$ evoluíram com complicação. As complicações mais comuns encontradas foram as infecções do tecido subcutâneo e deiscência da ferida operatória devido à infecção. Ocorreram ainda abscessos subfrênicos, pélvicos e intra-abdominais em $20 \%$ dos $\operatorname{casos}^{19}$.

Na República Dominicana foi realizado um estudo em pacientes submetidos à apendicectomias que demonstrou complicações em $5.2 \%$ dos pacientes. Em $47 \%$ destes pacientes foi necessária reintervenção, sendo a causa mais freqüente dela a infecção da ferida operatória. Nesta situação, a mortalidade foi $12.5 \%$ devido, principalmente, à septicemia ${ }^{20}$. Os resultados são diferentes em outro estudo realizado no México, onde as causas mais comuns de reoperações foram abscesso residual, oclusão intestinal, por bridas e evisceração ${ }^{21}$.

Percebe-se que as pesquisas e estatísticas encontradas em relação às complicações em pós-operatórios de apendicectomias são específicas para cada instituição analisada, e que por isso não há dados universais, o que leva a acreditar que os hospitais do Estado de Alagoas apresentam perfil próprio das complicações após apendicectomias.

Como a maior prevalência de complicações foram neste estudo no sexo masculino e nos extremos de idade deve a comunidade médica ficar alerta sobre os riscos maiores da apendicite aguda nesses indivíduos, fazendo-se necessários o diagnóstico e o tratamento precoce nesse grupo.

A dificuldade de acesso da população ao atendimento médico, aliado ao atraso no diagnóstico e tratamento da apendicite aguda - que estão associados à maior morbidade e maior número de complicações no pós-operatório -, pode justificar o que foi observado nos pacientes com mais de 4 dias de evolução neste grupo de pacientes.

\section{CONCLUSÕES}

Apesar da apendicite aguda se tratar de doença com tratamento cirúrgico considerado simples, foi encontrado neste estudo índice de complicações alto, podendo ser justificado pelo fato dele ter sido realizado em um hospital de emergência do Estado, onde as condições higieno-sanitárias são precárias. Contudo, mostrou-se que complicações nas apendicectomias foram mais freqüentes nos extremos de idade, quando houve atraso no diagnóstico e tratamento, e nas fases mais tardias da apendicite aguda. 
Nutels DBA, Andrade ACG, Rocha AC. Post-appendectomy complication profiling in an emergency hospital. ABCD Arq Bras Cir Dig 2007;20(3):146-9.

ABSTRACT - Background - Acute appendicitis is the most common and frequent acute surgical inflammatory lesion of the abdominal cavity. Aim - To analyse the most common post-operative complications of patients submitted to appendectomies. Methods - A retrospective analysis was performed using a research protocol, in patients submitted to appendectomies at Dr. Armando Lages' Emergency Unit, between the period of July 2005 through July 2006. Results - Complications occurred in 17\% of a total of 107 appendectomized patients. Thirteen patients (54\%) developed surgical infection wounds, followed by abdominal wall abscess in $12,5 \%$ and peritonitis in $8 \%$. zero point nine percent of the patients had to be re-operated and $0,9 \%$ died. Of the appendectomized patients 5,6\% were of preschool age, and of these, $33 \%$ developed complications. $10 \%$ of the complications occurred in patients of school age and adolescents and $20 \%$ occurred in young adults. Middle-aged adults and elderly patients, had $66 \%$ of complications. Of those who evolved in up to 24 hours of the disease, $17,2 \%$ had some sort of post-operative complication. Between 1 and 3 days, $15 \%$ and those with more than four days, 57,2\%. Conclusions - Appendectomy complications were more frequent at extreme ages, when there was a delay in diagnosis and treatment, as well as in more advanced phases of acute appendicitis.

HEADINGS - Appendicitis. Appendectomy. Postoperative complications.

\section{REFERÊNCIAS}

1. Addiss DG, Shaffer N, Foewler BS, Tauxe RV. The epidemiology of appendicitis and appendectomy in the United States. Am J Epidemiol. 1990;132:91025.

2. Anderson N, Griffiths H, Murphy J. Is appendicitis familial? Br Med J. 1979;2:697-8

3. Arnbjörnsson E. Acute appendicitis and dietary fiber. Arch Surg. 1983;118:86870 .

4. Aschoff L. Ueber die bedeutung des kotsteine in der aetiologie der epityphitis. Med Klin. 1931;24:587.

5. Attwood SE, Cafferkey MT, West AB. High appendicectomy rates in Ireland. J Epidemiol Community Health. 1987;41:72-3.

6. Barker DJP. Acute appendicitis and dietary fibre. Br J Med J. 1985;290:11257.

7. Barrera, C, et al. Complicaciones de la apendicectomia y tratamiento. Rev Cient Actual. 1997;12:61-2.

8. Burkitt DP. The etiology of appendicitis. Br J Surg. 1971;58:695-9.

9. Campo PR, et al. Complicaciones post-apendicectomia. Acta Med Domin. 1998. 20:184-7.

10. Cooperman M. Complications of appendectomy. Surg Clin North Am. 1983;63:1233-47.

11. Couto SO. Apendicite Aguda. In: Pires MTB, Starling SV, editores. Manual de urgências em pronto-socorro. $7^{\mathrm{a}}$ ed. Belo Horizonte: Medsi; 2002. p.34251.
12. Domínguez AV, et al. Reoperaciones en appendicitis. Rev Gastroenterol Méx. 1993;58:346-9.

13. León, LH, et al. Complicaciones locales de la apendicitis. Rev Gastroenterol Méx. 1994;59:223-6.

14. Neto JE, Celano, RM, Lanzoni, VF, et al. Apendicite aguda: modelo experimental em coelhos. Arq Gastroenterol. 2000;37:114-9.

15. Petrolanu A, Neto JE, Alberti LR. Comparative incidence of acute appendicitis in a mixed population, related to the skin color. Arq Gastroenterol. 2004;41:246.

16. Pieper R, Kager L, Nasman P. Acute appendicitis: a clinical study of 1018 cases of emergency appendectomy. Acta Chir Scand. 1982;148:51-4.

17. Silva JB, et al. Apendicite aguda na criança: experiência e estudo comparativo em apendicite supurada. Rev Bras Cir. 1985; 75:351-6.

18. Silva MW. Apendicite aguda. In: Neto JB, editor. Cirurgias de emergências: condutas. Rio de Janeiro: Revinter; 2000. p.344-6.

19. Van Zwalenburg C. Obstruction and consequent distention the cause of appendicitis. JAMA. 1904;42:820-7.

20. Wangensteen $\mathrm{OH}$, Bowers WF. Significance of the obstrutive factor in genesis of acute apendicitis. Arch Surg. 1937;34:496-504.

21. Wolkomir A, McGovern P. Seasonal variation of acute appendicitis. South Med J. 1987;80:958-60.

Conflito de interesse: não há

Fonte financiadora: não há

Recebido para publicação em: 20/04/2007

Aceito para publicação em: 15/05/2007 